\title{
Pregnancy in multiple sclerosis: clinical and self-report scales
}

\author{
R. F. Neuteboom - A. C. J. W. Janssens - T. A. M. Siepman - I. A. Hoppenbrouwers • \\ I. A. Ketelslegers - N. Jafari • E. A. P. Steegers - C. J. M. de Groot • \\ R. Q. Hintzen
}

Received: 29 June 2011/ Accepted: 12 July 2011/Published online: 3 August 2011

(C) The Author(s) 2011. This article is published with open access at Springerlink.com

\begin{abstract}
Relapse rate is decreased during pregnancy in multiple sclerosis (MS). Risk for postpartum relapse is increased in the first 3 months after delivery. We aimed to study clinical course of MS around pregnancy, using clinical as well as self-report scales, including data on quality of life (QoL), and to identify clinical factors predisposing for postpartum relapse. We performed a prospective, longitudinal study among 35 MS patients and 20 controls. In patients we assessed expanded disability status scale (EDSS), the Guy's neurological disability scale (GNDS) and the multiple sclerosis impact scale 29 (MSIS29). In patients and controls we assessed the MOS 36 item short form health survey questionnaire (SF36), consisting of eight domains. The previously described surge in relapses after delivery was also obvious in this study
\end{abstract}

R. F. Neuteboom - T. A. M. Siepman - I. A. Hoppenbrouwers ·

I. A. Ketelslegers · N. Jafari · R. Q. Hintzen $(\bowtie)$

Department of Neurology, Erasmus MC, Erasmus University

Medical Centre, Rotterdam, The Netherlands

e-mail: r.hintzen@erasmusmc.nl

R. F. Neuteboom - T. A. M. Siepman - I. A. Hoppenbrouwers · I. A. Ketelslegers · N. Jafari · R. Q. Hintzen

MS Centre ErasMS, Rotterdam, The Netherlands

A. C. J. W. Janssens

Department of Epidemiology, Erasmus MC, Erasmus University

Medical Centre, Rotterdam, The Netherlands

E. A. P. Steegers

Department of Obstetrics and Gynaecology, Erasmus MC,

Erasmus University Medical Centre, Rotterdam,

The Netherlands

C. J. M. de Groot

Department of Obstetrics and Gynaecology, VU Medical Centre, Amsterdam, The Netherlands $(p=0.005)$. At group level EDSS and MSIS-29 did not show overt fluctuations over time. The GNDS, however, improved during the third trimester, compared to the first trimester $(p=0.003)$. A concomitant improvement in the SF36 domains vitality $(p<0.001)$ and general health $(p=0.001)$ was found in patients. At the final visit, at least 9 months after delivery, no worsening of EDSS, GNDS, MSIS-29 or SF36 was observed compared with the (for MS, beneficial) third trimester. Duration of disease, relapses in the year preceding pregnancy or relapses during pregnancy were not associated with postpartum relapse. QoL is improved during pregnancy. Although relapse rate was increased directly after delivery, in the mid long term after delivery no adverse effects of pregnancy on MS were found.

Keywords Multiple sclerosis · Pregnancy ·

Quality of life · Postpartum relapse

\section{Introduction}

The clinical debut of multiple sclerosis (MS) in women is often during the childbearing years [1]. Factors associated with family planning are a major concern when having a chronic disease like MS. Pregnancy has a known ameliorating effect on the disease activity [2]. In the third trimester the relapse rate is decreased by around $70 \%$ compared to the year preceding pregnancy. Yet in the first 3 months after delivery, one in every three women has a relapse [2, 3].

Several questions arise when counselling women with MS who want to become pregnant. First, the timing of a postpartum relapse, early after delivery, may unfavourably influence daily care for the newborn infant. Prediction of a postpartum relapse would be most helpful in order to 
anticipate on this postpartum relapse. A previous study showed that the following three clinical factors were found to be predictive of a postpartum relapse: (1) the number of relapses in the year preceding pregnancy, (2) the number of relapses during pregnancy, and (3) the duration of disease [3]. These findings have not been validated in an independent prospective cohort.

Second, the clinical data in the few available studies on pregnancy and MS are limited to the expanded disability status scale (EDSS) and relapse rate. No quality of life $(\mathrm{QoL})$ issues or self report scales were addressed in these studies. Next to insight in disease course in terms of disability and attack frequency, also better insight in $\mathrm{QoL}$ during and after pregnancy is necessary to inform women with MS who want to become pregnant.

Third, it has been shown that the relapse rate declines after a peak in the first 3 months after delivery. Nine months after delivery the relapse rate is comparable with the relapse rate in the year preceding pregnancy. Whether QoL at that time has improved or worsened is unknown.

The aims of this prospective and longitudinal study were not only to confirm data on EDSS and relapse rate, but also to address clinical course and outcome by using clinical scales as well as self-report scales including QoL parameters. Our second goal was to identify or confirm clinical risk factors predisposing for postpartum relapse.

\section{Materials and methods}

\section{Participants and procedures}

The Rotterdam Study on Pregnancy in MS was performed at ErasMS, the MS Centre at Erasmus MC. Ambulant MS patients, with a relapsing remitting disease course, were invited to participate. Control patients were recruited from the out-patient clinic of Obstetrics at the Erasmus MC. Exclusion criteria for MS patients and controls were recurrent abortion, hypertension, diabetes mellitus or other systemic diseases. The study was designed to include patients preconceptionally, but inclusion was also allowed during pregnancy. Inclusion of all controls occurred during the first trimester. All MS patients and controls were seen at 10-12 weeks and 28-30 weeks of pregnancy, and at 4-8 weeks after delivery. MS patients were also invited at a time point at least 9 months after delivery, without clinical infection or recent disease activity.

Data on maternal age, disease duration, and parity were collected at the first visit. Data on gestational age at delivery, multiple pregnancy, (pre)eclampsia, delivery, birth weight, and breastfeeding were collected at the last two visits. Data on relapse rate before pregnancy, during the three trimesters of pregnancy, and every 3 months in the first year after delivery were collected by interview at all these time points. The Guy's neurological disability scale (GNDS), multiple sclerosis impact scale 29 (MSIS-29), and EDSS were assessed in patients at every visit. Both patients and healthy controls were asked to evaluate their health related QoL the month prior to evaluation using the MOS 36 item short form health survey questionnaire (SF36) at each visit.

This study was approved by the ethics committee of the Erasmus MC and all participants gave written informed consent.

\section{Measurements}

A relapse was defined as an episode of neurological disturbance for which causative lesions are likely to be inflammatory and demyelinating in nature and lasting more than $24 \mathrm{~h}$ [4]. Pseudo-relapse, meaning recurrence of previous signs and symptoms or fever-related worsening, was not considered as a relapse. Disease duration was measured from time of diagnosis.

The EDSS is the most widely used scoring system in MS and consists of findings of the neurological examination [5]. The EDSS is an ordinal score that ranges from 0.0 (no disability) to 10.0 (death due to MS). The EDSS was scored at every visit by trained neurologists (RFN, TAMS, IAH, IAK, NJ, and RQH).

The GNDS consists of 12 items, all generating a score ranging from 0 (optimal health) to 5 (poor health) with a summed maximum total score of 60 [6]. Items are cognition, mood, vision, speech, swallowing, lower and upper extremity function, bladder and bowel function, sexual function, fatigue, and one free item addressing another problem not yet contained in other items.

The MSIS-29 is a validated self-report scale consisting of two domains: physical health (20 items) and mental health (nine items) [7-9]. Both domains generate a score ranging from 0 (optimal health) to 100 (poor health). The questions reflected on the 2 weeks preceding evaluation.

The SF36 is an instrument comprising four physical health domains and four mental health domains and has been used before in MS patients [10-12]. The physical health domains are physical functioning, role physical functioning, bodily pain, and general health. The four mental health domains are social functioning, vitality, role emotional functioning, and mental health. For each domain a score is generated ranging from 0 (poor health) to 100 (optimal health).

Statistical analysis

Differences in demographic and pregnancy characteristics and the SF36 between patients and healthy women were compared using the Student's $t$ test or Mann-Whitney $U$ test 
for continuous variables and chi-square for categorical variables. The same statistics were used for comparing characteristics of MS patients with and without a postpartum relapse. Relapse rate, clinical scales and self-report scales at the different time points were compared to the third trimester using a multiple measurements linear mixed model analysis. The third trimester was used as reference because maximal disease amelioration has been reported before at that time point [2]. A $p$-value below 0.01 was considered significant. Data were analyzed using SPSS version 16.0.

\section{Results}

Characteristics of the study population

Thirty-five women with MS and 20 controls participated in this study. Eighteen MS patients were included before pregnancy, 16 during the first trimester, and one during the third trimester. The mean time from inclusion to pregnancy in the $18 \mathrm{MS}$ patients was 6 [standard deviation (SD): \pm 3.9 , range 1-20] months. The mean time from delivery to the final visit in the MS group was 10 (SD: \pm 3.5 , range 8-24) months. All controls were enrolled in the first trimester of pregnancy.

The controls were recruited from the outpatient clinic of Obstetrics in our hospital. The reasons for their referral were: previous cesarean section (six), history of cardiac problems (three, of which one also had twin pregnancy), medically assisted pregnancy (two), prematurity in previous pregnancy (two), previous post-natal depression (one), uterus duplex (one), epilepsy (one), twin pregnancy (one), metabolic disorder (one). Two women had no specific medical indication.

In the MS patients median disease duration was 4.0 (SD: \pm 4.0 , range 0-13) years. Median EDSS was 1.5 (SD: \pm 0.8 , range $0-4.0)$ in the first trimester $(n=34)$. Three MS patients received intravenous immunoglobulins directly after delivery with the aim to prevent possible relapses [13]. One woman had already received corticosteroids as treatment for a postpartum relapse at the time of her first postpartum visit. At time of the final visit four patients used immune modulating drugs.

Data on pregnancy and birth outcome are presented in Table 1. A smaller proportion of women were nulliparous in the control group, compared to the MS patients. Breastfeeding at time of the first postpartum visit was more frequently observed in the MS group, compared to the control group.

\section{Annualized relapse rate}

The annualized relapse rate is depicted in Fig. 1. After delivery a significantly increased relapse rate was observed
Table 1 Maternal age and data on pregnancy and birth outcome

\begin{tabular}{|c|c|c|}
\hline & $\begin{array}{l}\text { Patients } \\
N=35\end{array}$ & $\begin{array}{l}\text { Controls } \\
N=20\end{array}$ \\
\hline Maternal age $( \pm S D)$ (years) & $31.2( \pm 3.8)$ & $31.3( \pm 4.6)$ \\
\hline Nulliparous (\%) & 71 & 35 \\
\hline Cesarean section $(\%)$ & 14 & 25 \\
\hline $\begin{array}{l}\text { Assisted vaginal delivery (forceps/ } \\
\text { vacuum) }(\%)\end{array}$ & 11 & 5 \\
\hline Multiple (twin) pregnancy (\%) & 0 & 10 \\
\hline $\begin{array}{l}\text { Gestational age at delivery }( \pm \mathrm{SD}) \\
\text { (weeks) }\end{array}$ & $39.2( \pm 1.5)$ & $38.1( \pm 2.7)$ \\
\hline $\begin{array}{l}\text { Prematurity ( }<37 \text { weeks of gestation })^{\mathrm{a}} \\
(\%)\end{array}$ & 6 & 5 \\
\hline Birth weight $( \pm \mathrm{SD})$ (grams) $^{\mathrm{a}}$ & $\begin{array}{l}3,329 \\
\quad( \pm 354)\end{array}$ & $\begin{array}{l}3,496 \\
\quad( \pm 686)\end{array}$ \\
\hline Small for gestational age ${ }^{\mathrm{b}}(\%)$ & 0 & 0 \\
\hline Breast feeding $(\%)$ & 69 & 40 \\
\hline (pre)eclampsia (\%) & 0 & 0 \\
\hline
\end{tabular}

$M S$ Multiple Sclerosis, $S D$ standard deviation

${ }^{\text {a }}$ Not including twin pregnancy

b Small for gestational age is defined as birth weight under -2SD, using standardized intrauterine growth charts

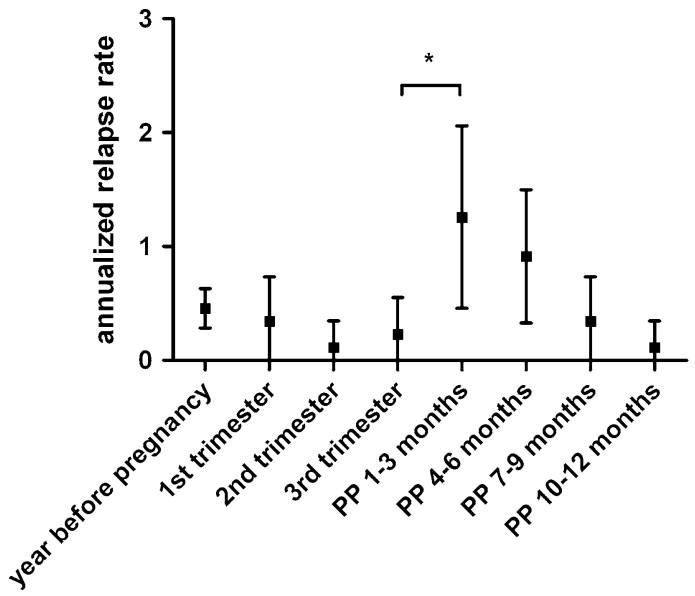

Fig. 1 Relapse rate: before, during and after pregnancy in MS patients. Values represent the mean $( \pm 95 \% \mathrm{CI})$ annualized relapse rate. The third trimester was used as reference. $* P$-value $<0.01 . P P$ postpartum

during the first 3 months after delivery compared to the third trimester $(p=0.005)$. There was no significant difference in the annualized relapse rate in the year preceding pregnancy compared to the period 9-12 months after delivery.

MS specific clinical and self report scales

The MS specific clinical and self report scales are shown in Fig. 2. The GNDS showed a significant drop during the 
Fig. 2 EDSS, GNDS and MSIS29 scores: before, during and after pregnancy in MS. Values represent mean $( \pm 95 \%$ CI) scores of the EDSS, GNDS, MSIS-29 (physical and psychological subscale). The third trimester was used as reference. ${ }^{*} P<0.01 . P P$ postpartum, EDSS expanded disability status scale, GNDS Guy's neurological disability scale, MSIS-29 multiple sclerosis impact scale 29
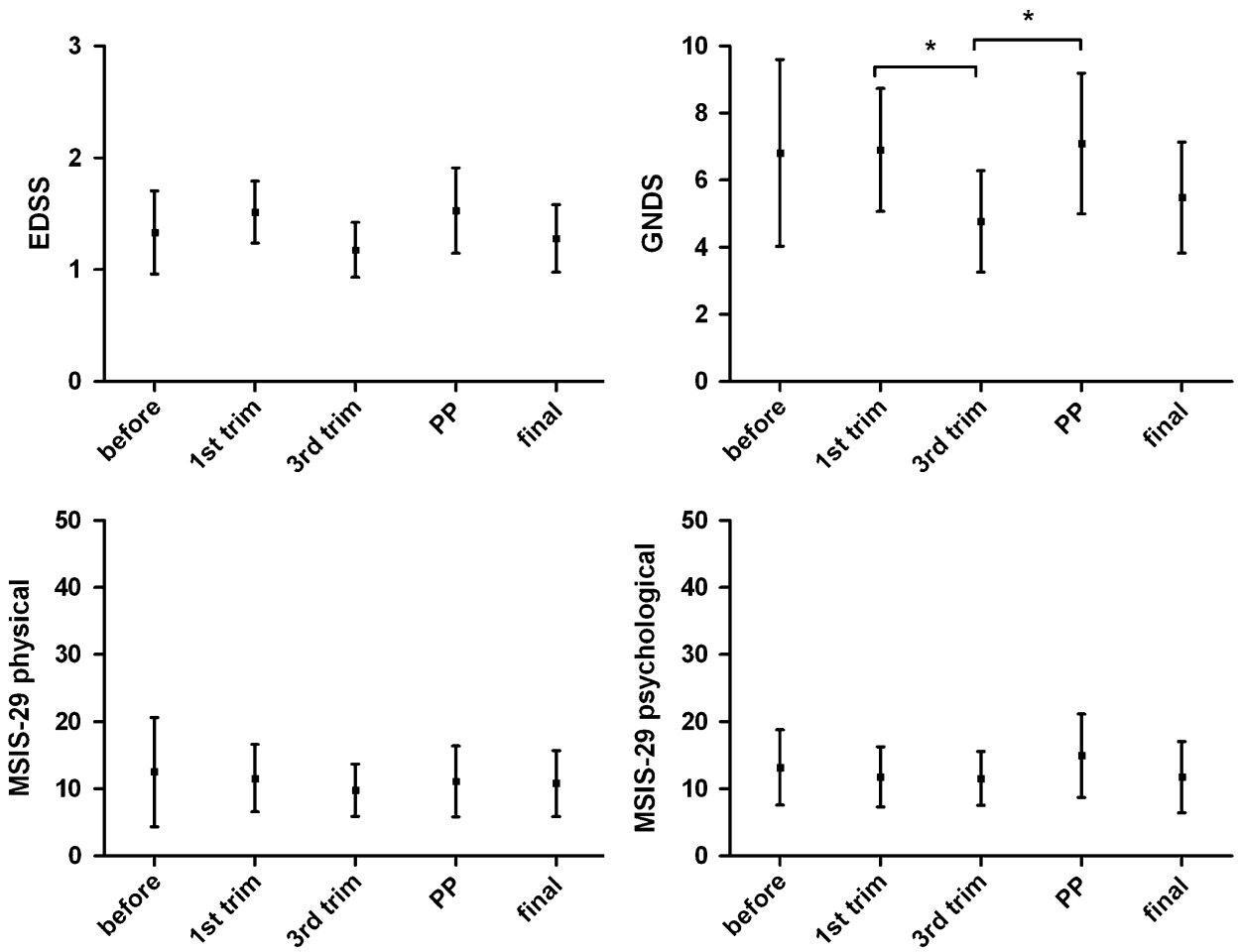

third trimester compared to the first trimester $(p=0.003)$ and the first postpartum visit $(p=0.001)$. We observed that about $85 \%$ of the mean drop in GNDS was mainly explained by three of the 12 items. These items concerned sexuality $(31 \%)$, fatigue $(28 \%)$, and a free item in which a complaint could be issued that was not addressed before in the other eleven items of the GNDS (26\%). No differences were observed in the two domains of the MSIS-29 or the EDSS during the study.

\section{Quality of life}

The SF36 was measured in both healthy controls and MS patients. The eight domains of the SF36 are shown in Fig. 3. MS patients reported an improvement in general health and in vitality during the third trimester compared to the first trimester (respectively, $p=0.001$ and $p<0.001$ ). Healthy women reported an evident and significant increase in bodily pain at time of the first postpartum visit ( $p=0.002)$ compared to the first trimester. The controls reported significantly more bodily pain than MS patients during the third trimester $(p<0.001)$. A significant decrease in physical functioning in MS patients was found during the third trimester, compared to the first trimester $(p=0.002)$. Physical functioning improved significantly after delivery in both MS patients $(p<0.001)$ and healthy women $(p<0.001)$. Role physical functioning was decreased in the controls during the third trimester compared the first postpartum visit $(p=0.009)$. No differences were found in the domains social functioning, role emotional functioning and mental health.

Postpartum relapse

Postpartum relapse occurred in 10 out of 35 MS patients $(29 \%)$. One out of the three women treated with intravenous immunoglobulins after delivery had a postpartum relapse. The differences between the patients with and without a postpartum relapse are shown in Table 2 . Women with a relapse during pregnancy were more likely to also experience a relapse after delivery. This difference was not significant. At the final visit, we found no differences in all assessment instruments, between patients with and without a postpartum relapse. Both groups also showed no differences in all assessment instruments comparing their third trimester visit with their final visit.

\section{Discussion}

We here present prospective and longitudinal data on clinical and QoL parameters during pregnancy in MS. We found an increase in relapse rate after delivery, comparable with previous studies $[2,3,14]$. We observed that this increase in relapse rate directly after delivery did not cause sustained adverse effects of pregnancy on MS in all used 
MS
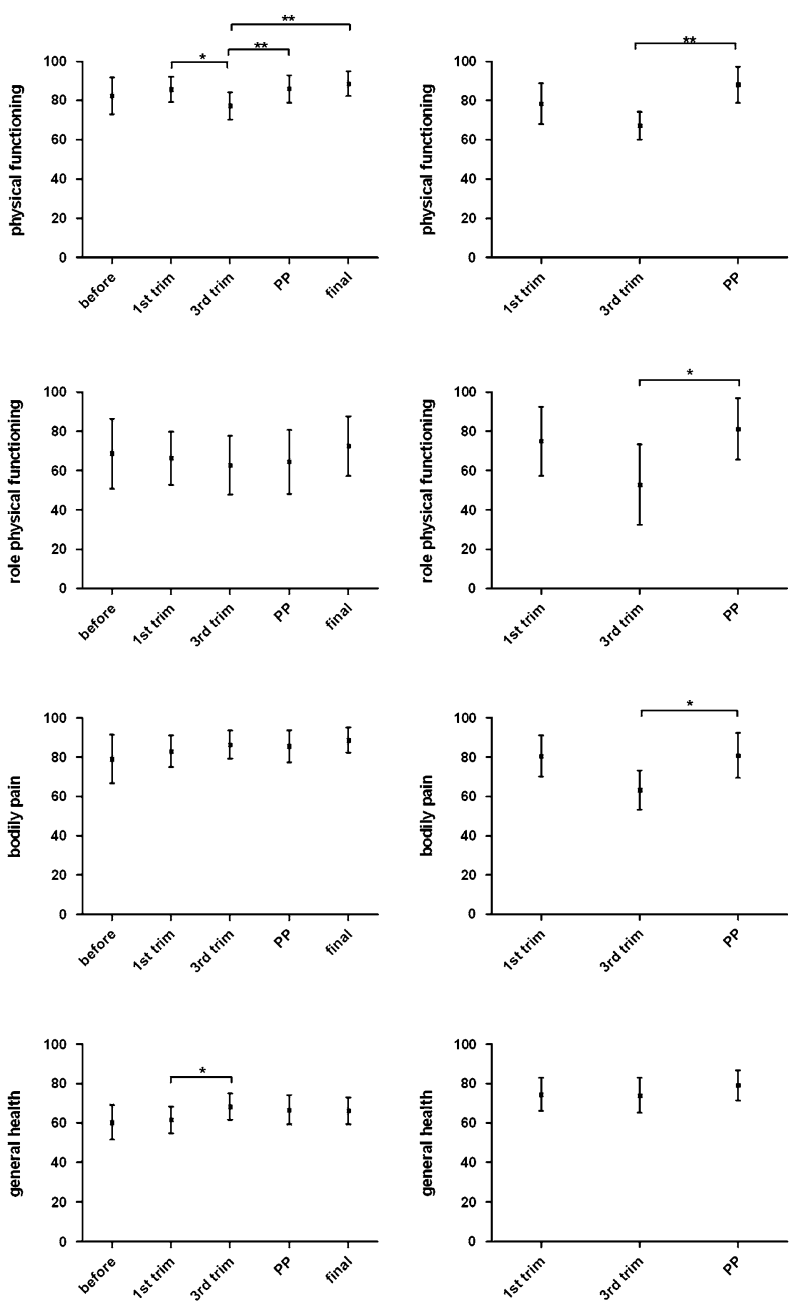

Fig. 3 SF36 subscale scores: before, during and after pregnancy in MS and healthy women. Values represent mean $( \pm 95 \% \mathrm{CI})$ scores on the eight subscales of the SF36: physical functioning, role physical functioning, bodily pain, general health, vitality, social functioning,
MS

Control
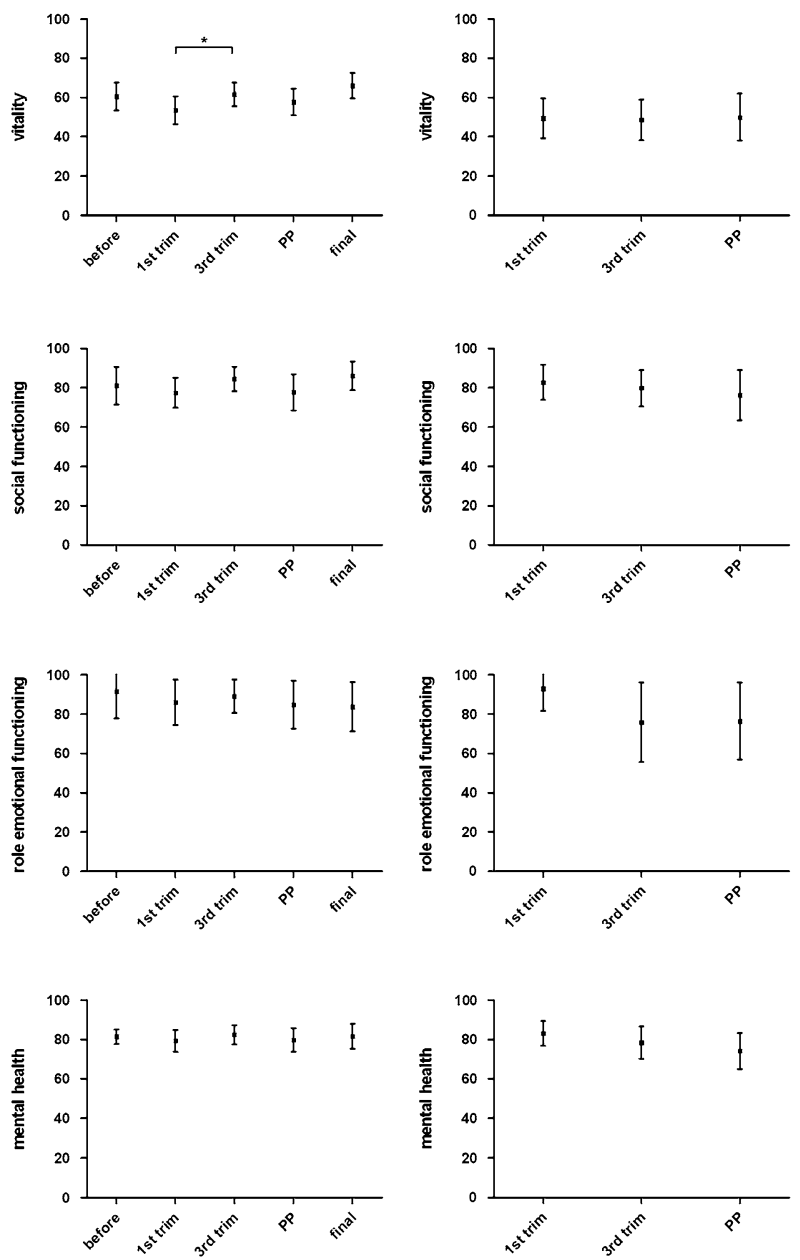

role emotional functioning and mental health. The third trimester was used as reference. Scores range from $0=$ poor health to $100=$ optimal health. ${ }^{*} P$-value $<0.01, * * P$-value $<0.001, P P$ postpartum, $S F 36$ MOS 36 item short form health survey questionnaire
Table 2 Characteristics of patients with and without postpartum relapse

$S D$ standard deviation, $P P$ postpartum, $I Q R$ interquartile range, EDSS expanded disability status scale

\begin{tabular}{llll}
\hline & $\begin{array}{l}\text { PP relapse } \\
N=10\end{array}$ & $\begin{array}{l}\text { No PP relapse } \\
N=25\end{array}$ & $P$ value \\
\hline Maternal age $( \pm \mathrm{SD})$ (years) & $31.1( \pm 4.5)$ & $31.3( \pm 3.6)$ & 0.76 \\
Disease duration $( \pm \mathrm{SD})$ (years) & $2.9( \pm 2.0)$ & $5.0( \pm 4.5)$ & 0.36 \\
Relapse in year before pregnancy $(\%)$ & 50 & 44 & 1.0 \\
Relapse during pregnancy (\%) & 30 & 8 & 0.13 \\
Nulliparous $(\%)$ & 70 & 72 & 1.0 \\
Median EDSS first trimester (IQR) & $2.0(1.5-2.13)$ & $1.25(1.0-1.88)$ & 0.01 \\
Assisted vaginal delivery or cesarean section $(\%)$ & 30 & 24 & 1.0 \\
\hline
\end{tabular}

instruments at time of the final visit, 9 months or more after delivery.

During pregnancy and after delivery we observed no difference in the MS specific assessment instruments EDSS and the self report scale MSIS-29. A small improvement in the GNDS was observed during the third trimester. Remarkably, this improvement in the GNDS was mainly explained by the domains concerning sexuality, fatigue, and 
the free item. Much in line with the decrease in fatigue is the improvement in the SF36 domains vitality and general health in MS patients during the third trimester. Contradictory to the amelioration of MS in the third trimester is the decreased health in the SF36 domain physical functioning in MS patients during the third trimester, compared with the first trimester and the first postpartum visit. On the other hand, decreased health in the SF36 domain physical functioning was also observed in controls during the third trimester compared to the postpartum visit. Therefore, this may be explained as a pregnancy effect and not MS related.

We also set out to validate several clinical risk factors for postpartum relapse that were previously identified [3]. These were disease duration, number of relapses in the year preceding pregnancy and number of relapses during pregnancy. We were not able to validate these findings in our cohort. We did observe that women with a relapse during pregnancy more likely also had a postpartum relapse, although this was not significant. It is possible that our numbers were too small to demonstrate such effects. Interestingly, a recent study showed that exclusive breastfeeding was associated with a smaller chance of postpartum relapse [17]. We and others could not reproduce these intriguing findings [2, 3, 14, 18, 19]. Taken together, we can conclude that, until now, there are no good clinical parameters predicting postpartum relapse. We, therefore, are in need of reliable biomarkers, able to predict a postpartum relapse. Interleukin 8 may be a candidate [20].

As described before $[15,16]$, we recorded no major adverse effects on birth outcome. A higher proportion of neonates small for gestational age in mothers with MS has been reported [15]. We also found a lower birth weight in the MS group, but this did not reach statistical significance.

Three points should be taken into account in respect to our data. First, the small numbers of patients and controls limited the conclusions of our study. We were not able to test for interaction effects and related measurements. In order not to make an over-interpretation we considered $p$-values under 0.01 to be significant and we used one visit as main reference. We chose the third trimester as main reference because maximal disease amelioration was reported at that time point $[2,3]$.

Second, the controls were referred to the outpatient clinic of Obstetrics mainly because of complications in previous pregnancies. Therefore, the chance of complications in the controls during these studied pregnancies and deliveries was increased. This may have influenced pregnancy outcome and QoL of the controls during and after pregnancy. It may likely explain why the controls reported more bodily pain than the women with MS during the third trimester.

Third, the participating patients in our study had a low EDSS, likely because we selected patients with a child wish. This low disability may have caused floor or ceiling effects limiting the ability of our measurement instruments to detect change. Especially the SF36 is sensitive to floor and ceiling effects [21]. It is possible that subtle differences in QoL were not detected by the SF36.

We can conclude that in the mid-long term after pregnancy in MS patients EDSS, GNDS, MSIS-29, relapse rate and QoL are not unfavourably altered. We also showed that amelioration of disease course during pregnancy is not only defined by a decreasing relapse rate, but can also be regarded in terms of QoL, mostly appreciated in the SF36 domains vitality and general health. This underlines the necessity for including assessments of QoL when determining MS disease severity. The results from this study will help neurologists and MS-nurses counselling pregnant MS patients or MS patients with a child wish.

Acknowledgments This Study was funded by the Dutch MS research foundation.

Conflict of interest The authors declare that they have no conflict of interest.

Open Access This article is distributed under the terms of the Creative Commons Attribution Noncommercial License which permits any noncommercial use, distribution, and reproduction in any medium, provided the original author(s) and source are credited.

\section{References}

1. Compston A, Coles A (2008) Multiple sclerosis. Lancet 372:1502-1517

2. Confavreux C, Hutchinson M, Hours MM, Cortinovis-Tourniaire P, Moreau T (1998) Rate of pregnancy-related relapse in multiple sclerosis. Pregnancy in multiple sclerosis group. N Engl J Med 339:285-291

3. Vukusic S, Hutchinson M, Hours M, Moreau T, CortinovisTourniaire P, Adeleine P, Confavreux C (2004) The Pregnancy in multiple sclerosis group; pregnancy in multiple sclerosis group. Pregnancy and multiple sclerosis (the PRIMS study): clinical predictors of post-partum relapse. Brain 127:1353-1360

4. Polman CH, Reingold SC, Edan G, Filippi M, Hartung HP, Kappos L, Lublin FD, Metz LM, McFarland HF, O'Connor PW, Sandberg-Wollheim M, Thompson AJ, Weinshenker BG, Wolinsky JS (2005) Diagnostic criteria for multiple sclerosis: 2005 revisions to the "McDonald Criteria". Ann Neurol 58:840-846

5. Kurtzke JF (1983) Rating neurological impairment in multiple sclerosis: an expanded disability status scale (EDSS). Neurology 33:1444-1452

6. Sharrack B, Hughes RAC (1999) The Guy's neurological disability scale (GNDS): a new disability measure for multiple sclerosis. Mult Scler 5:223-233

7. Hobart J, Lamping D, Fitzpatrick R, Riazi A, Thompson A (2001) The multiple sclerosis impact scale (MSIS-29) a new patientbased outcome measure. Brain 124:962-973

8. Riazi A, Hobart JC, Lamping DL, Fitzpatrick R, Thompson AJ (2002) Multiple sclerosis impact scale (MSIS-29): reliability and validity in hospital based samples. J Neurol Neurosurg Psychiatry 73:701-704 
9. Gray O, McDonnell G, Hawkins S (2009) Tried and tested: the psychometric properties of the multiple sclerosis impact scale (MSIS-29) in a population-based study. Mult Scler 15:75-80

10. Ware JE, Sherbourne CD (1992) The MOS 36-item short-form health survey (SF36). I. Conceptual framework and item selection. Med Care 30:473-483

11. Janssens AC, van Doorn PA, de Boer JB, Kalkers NF, van der Meche FG, Passchier J, Hintzen RQ (2003) Anxiety and depression influence the relation between disability status and quality of life in multiple sclerosis. Mult Scler 9:397-403

12. Janssens AC, van Doorn PA, de Boer JB, van der Meché FG, Passchier J, Hintzen RQ (2003) Impact of recently diagnosed multiple sclerosis on quality of life, anxiety, depression and distress of patients and partners. Acta Neurol Scand 108:389395

13. Haas J, Hommes OR (2007) A dose comparison study of IVIG in postpartum relapsing-remitting multiple sclerosis. Mult Scler 13:900-908

14. Fernández Liguori N, Klajn D, Acion L, Cáceres F, Calle A, Carrá A, Cristiano E, Deri N, Garcea O, Jaureguiberry A, Onaha P, Patrucco L, Riccio P, Rotta Escalante R, Saladino M, Sinay V, Tarulla A, Villa A (2009) Epidemiological characteristics of pregnancy, delivery, and birth outcome in women with multiple sclerosis in Argentina (EMEMAR study). Mult Scler $15: 555-562$
15. Dahl J, Myhr KM, Daltveit AK, Hoff JM, Gilhus NE (2005) Pregnancy, delivery, and birth outcome in women with multiple sclerosis. Neurology 65:1961-1963

16. Kelly VM, Nelson LM, Chakravarty EF (2009) Obstetric outcomes in women with multiple sclerosis and epilepsy. Neurology 73:1831-1836

17. Langer-Gould A, Huang SM, Gupta R, Leimpeter AD, Greenwood E, Albers KB, van den Eeden SK, Nelson LM (2009) Exclusive breastfeeding and the risk of postpartum relapses in women with multiple sclerosis. Arch Neurol 66:958-963

18. Neuteboom RF, Hintzen RQ (2011) Breast-feeding, postpartum and prepregnancy disease activity in multiple sclerosis. Neurology $76: 1532-1533$

19. Airas L, Jalkanen A, Alanen A, Pirttilä T, Marttila RJ (2010) Breast-feeding, postpartum and prepregnancy disease activity in multiple sclerosis. Neurology 75:474-476

20. Neuteboom RF, Verbraak E, Voerman JS, van Meurs M, Steegers EA, de Groot CJ, Laman JD, Hintzen RQ (2009) First trimester interleukin 8 levels are associated with postpartum relapse in multiple sclerosis. Mult Scler 15:1356-1358

21. Freeman JA, Hobart JC, Langdon DW, Thompson AJ (2000) Clinical appropriateness: a key factor in outcome measure selection: the 36 item short form health survey in multiple sclerosis. J Neurol Neurosurg Psychiatry 68:150-156 